\title{
An Evolving Public Health Crisis Caused by the Rapid Spread of the SARS-CoV-2 Delta Variant
}

\author{
The Protective Effect of Vaccination
}

\author{
Sohail Rao, MD, MA, DPhil ${ }^{1,2}$ and Manish Singh, MD, FACS ${ }^{3}$ \\ ${ }^{1}$ Executive Vice President, DHR Health, 5501 S. McColl Road, Edinburg, Texas 78539 and President \\ \& Chief Executive Officer, DHR Health Institute for Research \& Development, 5323 S. McColl Road, \\ Edinburg, Texas 78539. \\ ORCID: https://orcid.org/0000-0001-5027-9992 \\ ${ }^{2}$ Correspondence should be addressed to: Sohail Rao, MD, MA, DPhil., DHR Health Institute for \\ Research \& Development, 5323 S. McColl Road, Edinburg, Texas 78539. Tel: (956) 362-2387. E-mail: \\ s.rao@dhr-rgv.com \\ ${ }^{3}$ Chief Executive Officer, DHR Health, 5501 S. McColl Road, Edinburg Tx 78539 and DHR Health \\ Bariatric and Metabolic Institute, 500 Raphael Drive, Edinburg, TX 78539 \\ ORCID: Manish Singh: https://orcid.org/0000-0003-4146-3282
}

Received 06/24/2021

Accepted for publication 06/29/2021

Published 06/29/2021

Keywords: COVID-19; SARS-CoV-2; Delta Virus; Vaccination

SARS-CoV-2 virus has already infected over 181 million people worldwide and has resulted in the death of over 3.9 million (1). In the United States alone, over 33 million people have been infected with SARS-CoV-2 which has resulted in the death of over 600,000 (1). The rapid development and deployment of several effective vaccines against the virus has lowered but not eliminated the threat to imminent public health crisis. Further compounding this challenge is the continued vaccine hesitancy and the non-availability of vaccines globally (2).

The notable competence of viruses to adapt to new hosts and environments is largely conditional on their capacity to generate de novo multiplicity in a relatively short period of time (3). It is therefore predictable that the rates of spontaneous mutation would vary among viruses. Additionally, RNA viruses mutate at a much faster rate than DNA viruses and the size of the genome is conversely related to the rate of mutation. It is important to note that selective host and environmental pressures (such as the use of anti-viral agents, etc.) can impact the rate of viral mutation thus accelerating the generation of new and more contagious variants (4).SARS-CoV-2 is an RNA virus and under optimal conditions it has a very high rate of mutation. The Centers for Disease Control and Prevention has developed a variant classification scheme that defines three classes of SARS-CoV2 variants (5). To date, none of the SARS-CoV-2 variants has been identified as a Variant of High Consequence. On the contrary, seven Variant of Interest and six Variant of Concern have been identified (5). Most of the variants have mutations in the gene encoding SARS-CoV-2 spike protein, which affects their transmissibility; disease severity; reduced neutralization by antibodies generated against previous infection or vaccination; reduced efficacy of available treatment; and potential diagnostic impact.

One of the variants of SARS-CoV-2 that is a Variant of Concern and is rapidly spreading in the US is of the lineage B.1.617.2 (Figure 1). Erroneously labelled as the "Indian Variant", this mutant was first reported in India, but its true origin remains unknown (6). Renamed as the Delta variant by the World Health Organization, it is believed to be the cause of the devastating second wave of the pandemic in India, which crippled its healthcare infrastructure resulting in thousands of deaths everyday $(7,8)$. Also identified as the Variant of Global Concern, B.1.717.2 has been detected in more than 40 countries and is the dominant variant in the United Kingdom (9). The Delta variant has mutations in the gene encoding the spike protein causing substitutions T478K, P681R and 452R (10). Collectively, these mutations increase 
viral transmissibility and alter the neutralizing ability of monoclonal antibodies and convalescent plasma from recovered patients with COVID-19.

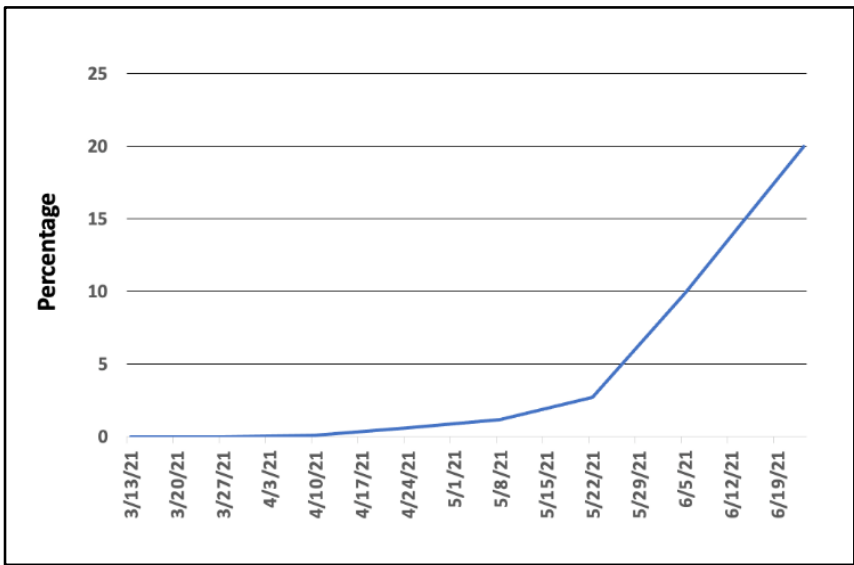

Figure 1: The percentage of Delta variants in patients diagnosed with SARS-CoV-2 in the US.

A recent study published in the journal Lancet suggested that the risk of hospitalization doubled in those infected with the Delta variant of SARS-CoV-2 as compared to the Alpha variant (11). Additionally, this study also underscored the protective effect of vaccination against infection with this variant. Pfizer-BioNTech vaccine was $88 \%$ effective against symptomatic disease from the B.1.617.2 variant two weeks after the second dose. Two doses of the AstraZeneca vaccine were $60 \%$ effective against symptomatic disease from the B.1.617.2 variant. It is notable that both vaccines were only $33 \%$ effective against symptomatic disease from Delta variant, three weeks after the first dose (11).

Given these observations, it can be envisaged that as in the past, SARS-CoV-2 virus will continue to mutate, and it is likely that future variants will be as infectious if not more than the currently circulating mutants. Therefore, it is imperative that we intensify our efforts to sequence all positive cases of COVID-19 to ascertain the extent of SARS-CoV-2 mutations. Lastly, given the protective effect of currently available vaccines, it is highly recommended that all qualified individuals 12 years and above get vaccinated to protect themselves from symptomatic disease resulting from exposure to either the wild-type and/or the variants of SARS-CoV-2 virus. Incentivizing qualified individuals should be seriously considered particularly in regions of relatively lower vaccination rates and younger adults who are seemingly hesitant to get vaccinated. Needless to say, a robust and sustained public health education program is essential to address existing crisis and to mitigate such challenges in the future.

\section{Diclosures}

SR declares no conflicts of interest.

MS declares no conflicts of interest.

\section{Funding}

The project is funded by a Seed Grant from the DHR Health Institute for Research \& Development

\section{References}

1. COVID-19 Dashboard - Johns Hopkins University Coronavirus Resource Center. June https://coronavirus.jhu.edu/map.html (Assessed Jun 28, 2021: 8:21 a.m. EST)

2. Killgore WDS., Cloonan SA, et al. The COVID-19 vaccine is here - Now who is willing to get it? Vaccines (Basel). 2021 Apr 1;9(4):339. doi: 10.3390/vaccines9040339. PMID: 33916161

3. Regoes RR., Hamblin S and Tanaka MM. Viral mutation rates: modelling the roles of within-host viral dynamics and the trade-off between replication fidelity and speed. Proc Biol Sci. 2013 Jan 7;280(1750):20122047. doi: 10.1098/rspb.2012.2047. Epub 2012 Nov 7.

4. Domingo E and Holland JJ. RNA virus mutations and fitness for survival. Annu Rev Microbiol. 1997;51:15178. doi: 10.1146/annurev.micro.51.1.151.

5. SARS-CoV-2 variant classifications and definitions. https://www.cdc.gov/coronavirus/2019ncov/variants/variant-info.html\#Interest. (Assessed Jun 23, 2021)

6. Cherian S., Agarwal A., et.al. Convergent evolution of SARS-CoV-2 spike mutations, L452R, E48Q and P681R, in the second wave of COVID-19 in Maharashtra, India. Available online: https://www.biorxiv.org/content/10.1101/2021.04.22.44 0932v1 (Assessed Jun 23, 2021)

7. Branswell $\mathrm{H}$. The name game for coronavirus variants just got easier. https://www.statnews.com/2021/05/31/the-name-gamefor-coronavirus-variants-just-got-a-little-easier/ (Assessed Jun 23, 2021)

8. Mudur GS. COVID: Second wave threatens to sink healthcare services. https://www.telegraphindia.com/india/second-wavethreatens-to-sink-healthcare-services-impact-of-covidbegins-to-tell-on-three-worst-affected-

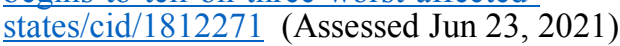

9. Delta variant causes more than $90 \%$ of new Covid cases in UK. https://www.theguardian.com/world/2021/jun/11/deltavariant-is-linked-to-90-of-covid-cases-in-uk (Assessed Jun 23, 2021) 
10. "Expert reaction to VUI-21APR-02/B.1.617.2 being classified by PHE as a variant of concern". sciencemediacentre.org. May 07, 2021. (Assessed Jun 23, 2021)

11. Sheikh A., McMenamin J., Taylor B., Robertson C., on behalf of Public Health Scotland and the EAVE II Collaborators. SARS-CoV-2 Delta VOC in Scotland: demographics, risk of hospital admission, and vaccine effectiveness. The Lancet. June 14, 2021. https://doi.org/10.1016/S0140-6736(21)01358-1 